\title{
2 ACOMPANHAMENTO FARMACOTERAPÊUTICO DE USUÁRIOS DE CARBONATO DE LítIO CADASTRADOS NO PROGRAMA DE SAÚdE MENTAL
}

\author{
| Nádia Martins'; Sheila Araújo²; Suelen de Souza³; Debora Corrêa ${ }^{4}$; Cassiano Saatkamp5; Régis Maestrii ${ }^{6}$ |
}

\section{RESUMO}

CONTEXTO: O Transtorno Bipolar do Humor é uma doença caracterizada por oscilações ou mudanças cíclicas de humor que pode causar grande impacto na vida do portador, de sua família e para a sociedade, gerando prejuízos para a saúde, reputação social e consequentemente sofrimento psicológico.

OBJETIVO: A pesquisa teve como objetivo analisar a litemia de pacientes em uso de carbonato de lítio.

METODOLOGIA: Pesquisa descritiva, de campo, prospectiva como abordagem quantitativa, dentre as variáveis investigadas, destacam-se: idade, sexo, escolaridade, estado civil, renda familiar, medicamentos prescritos e dosagem da litemia.

RESULTADOS: O perfil dos usuários caracterizou-se pelo sexo masculino 60\% (6/10), com idade média de 41,4 anos, 80\% (8/10) com renda familiar de 1 - 2 salários mínimos, 60\% tem ensino médio, 80\% (8/10) usam estabilizadores do humor associados à antipsicóticos, 80\% (8/10) estavam com a concentração de lítio abaixo da faixa terapêutica.

CONCLUSÕES: Esses resultados contribuem para o planejamento das ações voltadas ao serviço de saúde mental, assim como, a necessidade da implementação da Atenção Farmacêutica, na tentativa de buscar soluções para problemas relacionados a medicamentos, reconhecimento de interações medicamentosas e intervenções pela equipe multiprofissional em tempo hábil para melhorar a adesão ao tratamento medicamentoso proposto e consequentemente melhorar a qualidade de vida desses pacientes.

PALAVRAS-CHAVE: Assistência à saúde; Carbonato de lítio; Saúde mental

\section{RESUMEN}

"Seguimiento farmacoterapéutico de carbonato de litio usuarios registrados en el programa de salud mental"

CONTEXTO: El trastorno bipolar es una enfermedad caracterizada por fluctuaciones cíclicas o cambios de humor que puede causar ungran impacto enla vida del paciente, la familia y la sociedadella, generando perdidas menudo está em la salud, la reputación social y la angustia psicológica enconsecuencia.

OBJETIVO: Este estúdio tuvo como objetivo analizar El nivel de pacientes de lítio en carbonato de litio.

METODOLOGÍA: Campo de investigación descriptiva, enfoque prospectivo y cuantitativo, lãs principales variables investigadas fueron: edad, vie, educación, estado civil, ingresos familiares, los medicamentos conreceta y dosis de los niveles en sangre.

RESULTADOS: Se encontró que el perfil de los usuarios era: varón de $60 \%(06 / 10)$ con una edad media de 41,4 años, el $80 \%$ (8/10) conuningreso familiar de 1-2 vecesel salario mínimo, el $60 \%$ com la escuela secundaria grados $80 \%$ (8/10) estaban utilizando estabilizadores del estado de ánimo asociados com los antipsicóticos, $80 \%$ (8/10) tenía una concentración de litio por debajo del rango terapéutico.

CONCLUSIONES: Estos resultados contribuyen a la planificación de lãs acciones relacionadas com El servicio de salud mental, así como la necesidad de implementación de la atención farmacêutica e nun intento de encontrar soluciones para: problemas relacionados com las drogas, elreconocimiento de lãs interacciones entre medicamentos y las intervenciones por equipo multidisciplinario equipo para mejorar la adherencia al tratamiento farmacológico propuesto y em consecuencia mejorar la calidad de vida de estos pacientes.

\section{DESCRIPTORES: Prestación de atención de salud; Carbonato de lítio; Salud mental}

\begin{abstract}
"Pharmacotherapeutic follow-up of lithium carbonate registered users in the mental health program"

BACKGROUND: Bipolar disorder is a disease characterized by fluctuations or cyclical mood swings that can cause great impact on the lives the patient, her family and society, generating often losses for in health, social reputation and consequently psychological distress.

AIM: This study aimed to analyze the lithium level of patients on lithium carbonate.

METHODS: Descriptive research field, prospective and quantitative approach, the main variables investigated were: age, sex, education, marital status, family income, prescription drugs and dosage of blood levels.

RESULTS: It was found that the users profile was: male $60 \%(6 / 10)$ with a mean age of 41.4 years, $80 \%$ (8/10) with family income $1-2$ times the minimum wage, $60 \%$ with high school degrees $80 \%$ (8/10) were using mood stabilizers associated with antipsychotics, $80 \%(8 / 10)$ had a lithium concentration below the therapeutic range.

CONCLUSIONS: These results contribute to the planning of actions related to the mental health service, as well as the need for implementation of pharmaceutical care in an attempt to find solutions for: drug-related problems, recognition of drug interactions and interventions by multidisciplinary team in time to improve the adherence to the proposed drug treatment and consequently improve the quality of life of these patients.
\end{abstract}

\section{KEYWORDS: Delivery of health care; Lithium carbonate; Men- tal health}

Submetido em 15-12-2015

Aceite em 30-01-2017

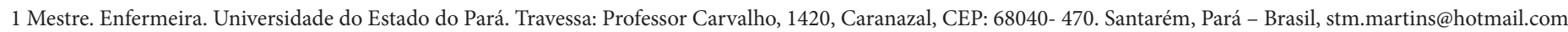
2 Graduada. Farmacêutica. Drogaria Planalto. Travessa: Mocidade, 70, Diamantino, CEP: 68020-165. Santarém, Pará - Brasil, limasheila31@hotmail.com.

3 Graduada. Farmacêutica. Fundação Esperança. Avenida: Mendonça Furtado, 2400, Mapiri, CEP: 68040-050. Santarém, Pará - Brasil, s.s.souza@hotmail.com

4 Acadêmica de Farmácia. Instituto Esperança de Ensino Superior. Travessa: Professor Carvalho, Caranazal, CEP: Santarém, Pará - Brasil, deb.carol15@hotmail.com

5 Mestre. Farmacêutico. Instituto Esperança de Ensino Superior. Rua Quixada, 21, Esperança, CEP: 68030- 250. Santarém, Pará - Brasil, Juniorsc7@yahoo.com.br

6 Mestre. Farmacêutico. Instituto Esperança de Ensino Superior. Avenida: Frei Vicente, 690, Aeroporto Velho, CEP: Santarém, Pará - Brasil, regismaetri@hotmail.com
}

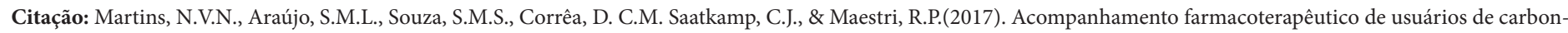
ato de lítio cadastrados no programa de saúde mental. Revista Portuguesa de Enfermagem de Saúde Mental (17), 09-16. 


\section{INTRODUÇÃO}

O Transtorno Bipolar do Humor, antigamente denominado de psicose maníaco depressiva, é caracterizado por oscilações ou mudanças cíclicas de humor. Estas mudanças vão desde oscilações normais, como nos estados de alegria e tristeza, até mudanças patológicas acentuadas e diferentes do normal, como episódios de mania, hipomania, depressão e mistos (Figueiredo, Souza, Dell'Áglio, \& Argimon, 2009).

A base da causa para a doença bipolar do humor não é inteiramente conhecida, os fatores genéticos, sociais e psicológicos somam-se no desencadeamento da doença. Em geral, os fatores genéticos podem determinar como o indivíduo reage aos estressores psicológicos e sociais, mantendo a normalidade ou desencadeando a doença (Gajwani, 2009).

A prevalência do Transtorno Bipolar (TB) tipo I é de cerca de $1 \%$ na população mundial, e não há diferenças entre o sexo masculino e feminino (Belmaker, 2004). Já o TB tipo II é mais prevalente no sexo feminino, afetando entre 1,1 e $5 \%$ da população mundial (Benazzi, 2007). Aproximadamente $54 \%$ dos pacientes apresentam episódio depressivo inicial, e a idade média de início dos sintomas ocorre predominantemente na faixa entre 15 e 19 anos (Kupfer et al, 2002).

A grande maioria dos pacientes com TB apresentam alguma comorbidade psiquiátrica. As principais são: abuso de álcool (30 a 69\%), abuso de substâncias (14 a $60 \%$ ), transtornos ansiosos (49 a 92\%), transtorno obsessivo-compulsivo (21\%), transtorno de pânico $(20,8 \%)$ e transtornos de personalidade (29 a 38\%) (Krishnan, 2005). As taxas de comorbidades clínicas entre os pacientes com TB também são elevadas. Em um estudo de Beyer, Kuchibhatla, Gersing \& Krishnan (2008) com 1.379 pacientes bipolares tipo I com idade média de 32,8 anos, mostrou que $44 \%$ dos pacientes apresentaram alguma comorbidade clínica. Guo, Keck, Li, Jang \& Kelton (2008) realizaram uma coorte retrospectiva envolvendo 67.862 pacientes com diagnóstico de TB ou ciclotimia. Observaram prevalência de diabetes mellitus de 7,7\%, hipertensão arterial sistêmica de $19,1 \%$, doença pulmonar obstrutiva crônica de $3,4 \%$, doença cardiovascular de $2,6 \%$, artrite reumatoide de $2,5 \%$ e obesidade de $6,2 \%$ em pacientes com TB.

Atualmente o lítio é usado para tratar mania, sendo efetivo em 60 a 80\% de todos os episódios de humor (Craig \& Stitzel, 2005). Foi o primeiro fármaco aprovado pela FDA (Food Drug Administration), para o Tratamento de Bipolar, sendo utilizado há mais de quarenta anos para o tratamento da doença Bipolar. É o único medicamento com propriedades estabilizadoras do humor comprovadas (Rosa, 2006).

O lítio é usado como monoterapia ou associado a outras drogas, como medicamento no manejo de episódios agudos de mania e depressão no TB I e II (Dourado, 2013).

O lítio é excretado quase inteiramente pelos rins, com meia-vida entre 14 e 30 horas. As preparações convencionais podem ser administradas em duas tomadas diárias, podendo, as de liberação lenta ser utilizadas em dose única (nesses casos, os níveis séricos matinais estarão aumentados em até 30\%). Usando-se doses maiores, mesmo o preparado de liberação lenta é mais comumente utilizado em duas tomadas diárias.

Segundo Vismari (2002) há uma tendência na diminuição dos níveis séricos de lítio recomendados ao longo das décadas, desde a primeira introdução do lítio, onde nas litemias acima de $0,8 \mathrm{mEq} / \mathrm{L}$ os efeitos colaterais superam os terapêuticos. A dosagem do lítio deve ser feita sempre 12 horas após a última ingestão do comprimido de lítio. Em geral, dosam-se os níveis séricos de cinco a sete dias após o início do tratamento, quando o lítio atinge seu steady state (curso estável), depois a cada sete ou quatorze dias, passando-se ao controle posterior a cada dois ou três meses, nos primeiros seis meses, e a cada quatro ou, no mínimo, a cada seis meses, posteriormente. Os níveis recomendados ao tratamento situam-se entre $0,6 \mathrm{mEq} / \mathrm{L}$ a $1,2 \mathrm{mEq} / \mathrm{L}$. O teto mais alto $(1,2 \mathrm{mEq} / \mathrm{L})$ é reservado para os estados agudos. Para a manutenção, as doses entre $0,6 \mathrm{mEq} / \mathrm{L}$ e $0,8 \mathrm{mEq} / \mathrm{L}$ são geralmente suficientes, procurando-se manter o paciente com as menores doses necessárias para a profilaxia (alguns pacientes apresentam resultados satisfatórios com $0,4 \mathrm{mEq} / \mathrm{L}$, já outros, eventualmente, precisam de doses maiores do que $0,8 \mathrm{mEq} / \mathrm{L}$ ). Em geral, inicia-se o tratamento com $300 \mathrm{mg}$ de carbonato de lítio à noite, aumentando-se as doses gradativamente até alcançar os níveis séricos desejados, levando-se em conta a tolerabilidade do paciente aos efeitos colaterais, o lítio é excretado quase inteiramente pelos rins, com meia-vida entre 14 e 30 horas (Goodman \& Gilman, 2006).

O conceito de Problemas Relacionados aos Medicamentos (PRM) encontra-se definido no Segundo Consenso de Granada como: problemas de saúde, entendidos como resultados clínicos negativos, derivados do tratamento farmacológico, que, produzidos por diversas causas, têm como consequência o não alcance do 
objetivo terapêutico desejado ou o aparecimento de efeitos indesejáveis (Santos, 2004).

É definida como suspeita de PRM a situação em que o doente está em risco de sofrer de um problema de saúde associado ao uso de medicamentos, geralmente devido à existência de um ou mais PRMs. Sendo os PRMs são todas aquelas situações, que durante o processo de utilização dos medicamentos, podem causar o aparecimento de um Resultado Negativo associado à Medicação (De Meneses e Sá, 2010).

Levando em consideração que a Atenção Básica em saúde é a porta de entrada para pessoas com problemas psíquicos e / ou transtorno mental instalado e que, a inexistência de um componente primordial no acolhimento deste usuário com sofrimento psicológico associado a toda e qualquer doença, às vezes atua como empecilho à adesão a práticas preventivas e, que devem ser usados todos os recursos terapêuticos ao alcance da equipe multiprofissional para minimizar a dor e o sofrimento dos mesmos, destacamos que é de fundamental importância conhecer o perfil demográfico, socioeconômico e clínico dos pacientes do programa de saúde mental, mais especificamente os que utilizam o carbonato de lítio em seu plano farmacoterapêutico.

Portanto este estudo justifica-se pela necessidade de se obter um conhecimento específico desta clientela com objetivo geral de analisar a litemia de pacientes em uso de carbonato de lítio atendidos na Unidade Básica de Saúde de Aparecida / Caranazal, no município de Santarém, Estado do Pará.

\section{MÉTODOS}

A pesquisa é classificada como descritiva, prospectiva, de campo, com abordagem quantitativa, de perspectiva local, realizada na Unidade Básica de Saúde (UBS) Aparecida / Caranazal, localizado na Av. Magalhães Barata, no 995, bairro Aparecida, na zona urbana do município de Santarém, Estado do Pará, a coleta de dados ocorreu no período previsto no projeto de pesquisa que foi de dezembro de 2013 a março de 2014.

A população alvo consistiu em pacientes cadastrados no Programa de Saúde Mental da UBS residentes na cidade de Santarém, Estado do Pará e que no momento do estudo faziam uso de carbonato de lítio em sua terapia medicamentosa.

Na UBS foi realizado um levantamento em livros de registro e de dispensação de medicamentos controlados (Portaria n. ${ }^{\circ} 344$, de 12 de maio de 1998) do quantitativo de usuários de carbonato de lítio, chegou-se a um número de 17 pacientes que após realização de visitas domiciliares a amostra para o estudo foi definida em 10 pacientes.

Foram incluídos na pesquisa pacientes cadastrados e em acompanhamento na UBS Aparecida / Caranazal no Programa de Saúde Mental, que faziam uso de Carbonato de lítio como parte do tratamento medicamentoso, pacientes de ambos os sexos e maiores de 18 anos de idade.

Foram excluídos da pesquisa os prontuários de pacientes que por qualquer motivo estavam faltosos no programa há mais de 01 ano e / ou não continham as informações necessárias sobre as variáveis pesquisadas, pacientes com idade inferior a 18 anos e os que não utilizavam o carbonato de lítio como parte da terapia medicamentosa.

É válido destacar que não houve a possibilidade de se trabalhar com uma amostra representativa dos pacientes com THB residentes no município de Santarém, Estado do Pará, a amostra desde estudo é referente a apenas uma (01) UBS, sendo caracterizada como uma amostra não representativa para a população geral do município de Santarém. Portanto, não houve a preocupação de generalizar os resultados, mas sim trabalhar com indicativo de tendência.

Posteriormente foi realizada uma abordagem analítica, descritiva nos prontuários para recolhimento de informações relacionadas às variáveis pesquisadas. A partir disso e seguindo as orientações do programa de saúde mental instituído pelo Ministério da Saúde foi analisado o resultado do exame dos pacientes em uso de carbonato de lítio para averiguação da litemia (dosagem de lítio) registrado nos prontuários.

Em seguida foram realizadas visitas domiciliares para orientação dos pacientes e familiares sobre os objetivos da pesquisa, foram detalhadas as etapas de acompanhamento dos pacientes por um período de três (03) meses e aplicação do Termo de Consentimento Livre e Esclarecido (TCLE). Após a assinatura do TCLE, durante o estipulado para o acompanhamento nas visitas domiciliares, os participantes da pesquisa foram orientados sobre o seu tratamento farmacológico, cuidados com a saúde e realização da dosagem sérica de lítio.

A pesquisa em questão teve uma metodologia baseada em aplicação de questionário redigido em papel que foi realizado por meio de visita domiciliar o que por ventura poderia ocasionar constrangimento em sua abordagem, fato que foi totalmente minimizado pelo preparo técnico dos pesquisadores, e ainda a adoção de um 
protocolo de armazenagem dos documentos em local seguro e sem a identificação das pessoas entrevistadas, no entanto, caso o participante se sentisse constrangido poderia desistir a qualquer momento da participação na pesquisa. Em caso de dano pessoal, diretamente provocado pelos procedimentos propostos pelos pesquisadores, os participantes tiveram assegurados à garantia de indenizações legalmente estabelecidas, conforme as leis vigentes no país.

Esta pesquisa seguiu todas as orientações da resolução 466/12 do Conselho Nacional de Saúde que descreve todas as normas de Pesquisas Envolvendo Seres Humanos, foi submetida, avaliada pelo Comitê de Ética e Pesquisa da Universidade do Estado do Pará e aprovada sob o parecer de número 490.728 de 12 de dezembro de 2013.

Foi realizado um levantamento de informações nos livros de registro da UBS Aparecida / Caranazal e elaborado um banco de dados em planilhas construídas com auxílio dos programas EXCEL e WORD da Microsoft 2010, e após análise os resultados foram apresentados através de uma estatística descritiva em forma de distribuição e porcentagem através de tabelas e gráficos.

\section{RESULTADOS}

Foram avaliados nesta pesquisa 10 pacientes com diagnóstico inicial de Transtorno Bipolar do Humor (THB) acompanhados em uma UBS e em tratamento contínuo. Na Tabela 1 destacam-se as características sócio demográficas dos pacientes estudados. A média de idade dos pacientes foi de 41,4 anos. A prevalência em relação ao sexo foi de $60 \%(6 / 10)$ para o sexo masculino. Com relação ao nível de escolaridade, foi observado no estudo que a maioria, $60 \%$ (6/10) dos pacientes pesquisados relataram ter completado o ensino médio. Porém, é válido destacar que 10\% (1/10) declarou ter completado o ensino superior. Relacionado ao estado civil entre os estudados se evidenciou a prevalência de solteiros $90,0 \%(9 / 10)$.
Tabela 1 - Características sócio demográficas de pacientes em uso de carbonato de lítio atendidos na Unidade Básica de Saúde Aparecida / Caranazal.

\begin{tabular}{|c|c|c|}
\hline VARIÁVEIS & $(n=10)$ & $\%$ \\
\hline \multicolumn{3}{|l|}{ Gênero } \\
\hline Masculino & 06 & 60 \\
\hline Feminino & 04 & 40 \\
\hline \multicolumn{3}{|l|}{ Faixa etária } \\
\hline $18-\mid 30$ & 01 & 10 \\
\hline $31-40$ & 04 & 40 \\
\hline $41-\mid 50$ & 04 & 40 \\
\hline 51 ou mais & 01 & 10 \\
\hline \multicolumn{3}{|l|}{ Escolaridade } \\
\hline Não alfabetizado & 02 & 20 \\
\hline Ensino médio incompleto & 01 & 10 \\
\hline Ensino médio completo & 06 & 60 \\
\hline Ensino superior & 01 & 10 \\
\hline \multicolumn{3}{|l|}{ Estado civil } \\
\hline Casado & 01 & 10 \\
\hline Solteiro & 09 & 90 \\
\hline \multicolumn{3}{|l|}{ Ocupação } \\
\hline Aposentado & 05 & 50 \\
\hline Trabalhador informal & 01 & 10 \\
\hline Desempregado & 04 & 40 \\
\hline \multicolumn{3}{|l|}{ Renda Familiar } \\
\hline 1 a 2 salários mínimos & 08 & 80 \\
\hline 3 a 4 salários mínimos & 02 & 20 \\
\hline
\end{tabular}

Fonte: Dados da pesquisa

Na Tabela 2 estão representadas às características clínicas. Nos resultados da presente pesquisa mostra-se que $60 \%$ dos pesquisados não informaram possuir doenças prévias ao diagnostico de THB, porém no diagnóstico atual, associados ao THB destacam-se a depressão em $31,58 \%(6 / 10)$ dos pesquisados e a esquizofrenia em $15,79 \%$ dos casos (3/10).

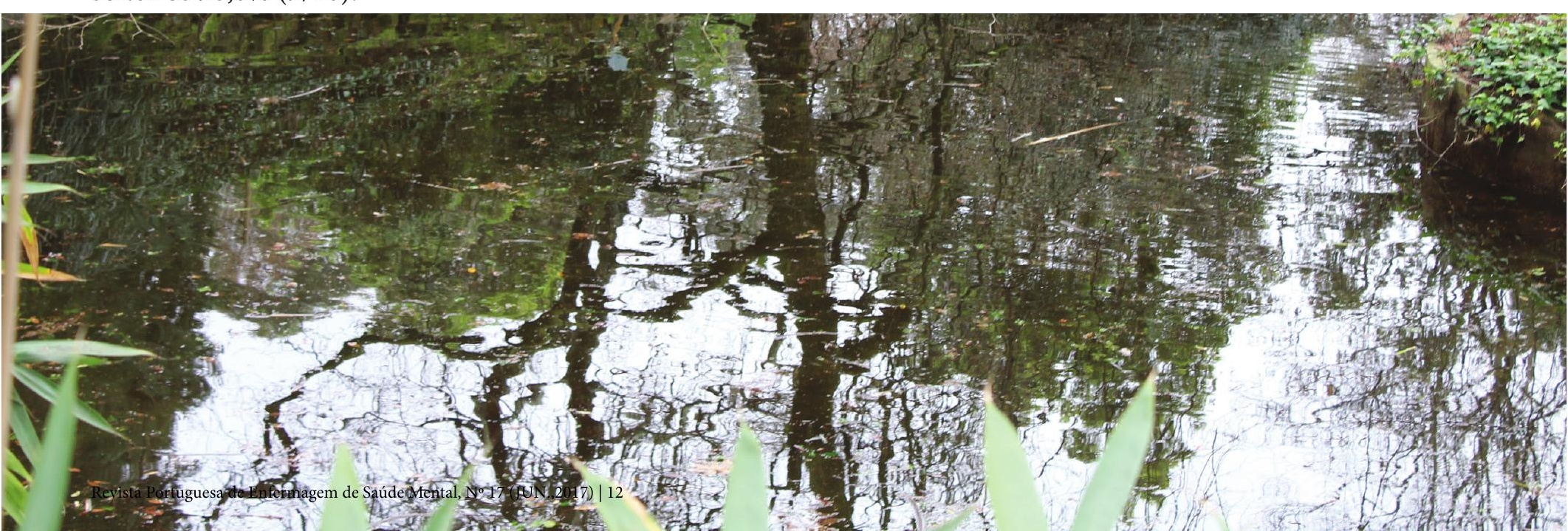


Tabela 2 - Características clínicas de pacientes em uso de carbonato de lítio atendidos na Unidade

Básica de Saúde Aparecida / Caranazal

\begin{tabular}{|l|c|c|}
\hline VARIÁVEIS & $(\mathrm{n}=10)$ & $\%$ \\
\hline Problemas de saúde anterior & 06 & 60 \\
\hline Não possuíam & 01 & 10 \\
\hline Álcool & 01 & 10 \\
\hline Déficit Visual & 01 & 10 \\
\hline Diabetes & 01 & 10 \\
\hline Outros & 03 & 17,65 \\
\hline Profissional consultado durante o acompanhamento do THB \\
\hline Neurologista & 01 & 5,88 \\
\hline Clinico geral & 06 & 35,29 \\
\hline Enfermeiro & 07 & 41,18 \\
\hline Psiquiatra & 01 & 10 \\
\hline Diagnóstico atual & 06 & 60 \\
\hline Transtorno do humor & 03 & 30 \\
\hline Transtorno do humor / Depressão &
\end{tabular}

Fonte: Dados da pesquisa

Durante o desenvolvimento da pesquisa no momento do acompanhamento farmacoterapêutico ficou evidenciado alguns dos possíveis motivos que podem levar a baixa adesão ao tratamento, levando ao comprometimento da terapêutica, o que pode ser evidenciado ao se analisar os resultados de litemia, alguns desses problemas pode está intrínseco ao Sistema Único de Saúde (SUS) que não disponibiliza profissionais médicos especializados na área da saúde mental em número suficiente para o suprimento da demanda nos serviços de referência para os atendimentos de forma satisfatória; outro problema que pode ser apontado é a baixa oferta de exames complementares como a dosagem de lítio, avaliação renal e hepática, o que implica na demora em retorno do paciente com o profissional médico para ser reavaliado; a oferta insuficiente de medicamentos necessários ao tratamento; a falta do profissional farmacêutico nas Unidades Básicas de Saúde, dentre outros motivos.

A monitorização periódica do lítio por dosagem sérica ou eritrocitária e por escalas de adesão em pacientes bipolares tem como objetivos: 1) avaliar a eficácia da terapêutica instituída; 2) monitorar a adesão ao tratamento prescrito do ponto de vista de doses e intervalos corretos e continuar a encorajar o uso durante o tratamento de manutenção; 3) determinar e ajustar as diferenças farmacocinéticas entre os indivíduos e no mesmo indivíduo, com relação a efeitos adversos e uso associado de outros medicamentos.
Nesse sentido, a monitorização terapêutica do lítio e de adesão ao tratamento com essa droga é útil para diagnóstico de tratamentos subterapêuticos, individualização da dose ou ajuste da dose terapêutica efetiva (Rosa et al, 2006).

Apresentados na Figura 1 estão os resultados a dosagem de lítio realizada pelos pacientes em acompanhamento no período da pesquisa, é importante destacar que dos 10 pacientes, apenas 01 não realizou os exames propostos por motivo pessoal de não aceitação e até mesmo por manifestar atitudes de agressividade e reclusão em domicílio, fato que reforça a importância do acompanhamento farmacoterapêutico e ainda a monitorização do lítio para determinação do tratamento adequado. Portanto dos 09 pacientes que realizaram a dosagem de litemia 11,1\% (01/09) está acima da faixa terapêutica caracterizado como risco para intoxicação, 11,11\% $(01 / 09)$ estavam dentro da faixa terapêutica podendo ser considerado com boa adesão ao tratamento e 77,78 $\%$ estavam abaixo da faixa terapêutica caracterizando que nos pacientes estudados o tratamento pode está sendo ineficaz.

Figura 1 - Resultados de litemia e avaliação da faixa terapêutica de pacientes em uso de carbonato de lítio na Unidade Básica de Saúde Aparecida / Caranazal

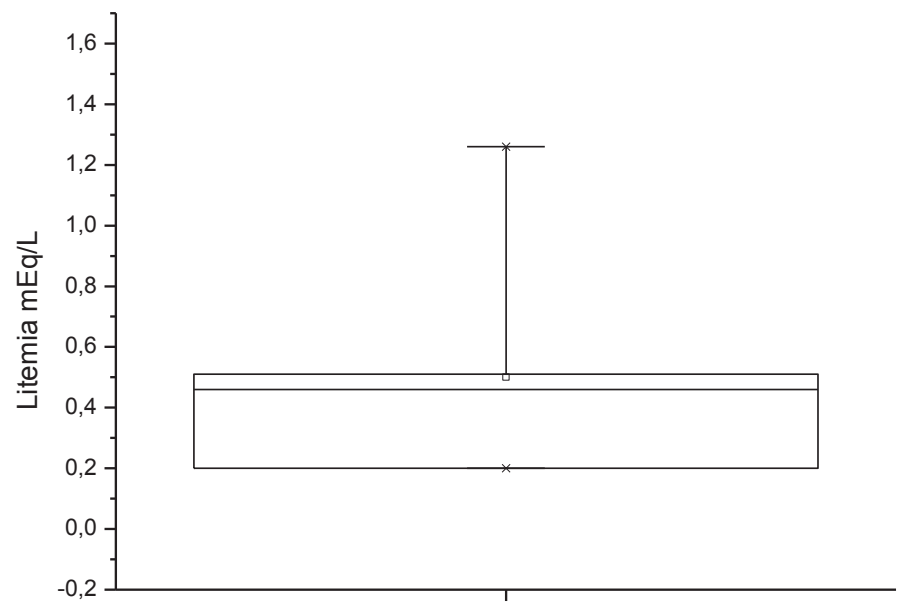

Pacientes em uso de carbonato de lítio

Fonte: Dados da pesquisa

Para o acompanhamento farmacoterapêutico, foi realizada a metodologia de DÁDER. A avaliação dos Problemas Relacionados a Medicamentos (PRM's) seguiu a classificação do Segundo Consenso de Granada (Comité de Consenso, 2002). A partir de uma análise focada no processo de uso do medicamento, constatou-se que cada paciente apresenta pelo menos um tipo de PRM. 
Demostrado na Tabela 3 estão os tipos de Problemas Relacionados a Medicamentos encontrados nos pacientes acompanhados onde $10 \%(1 / 10)$ foi classificado quanto a necessidade em PRM 02, que destaca efeito de um medicamento não necessário, tendo por base para essa determinação, o relato do paciente em manter a necessidade humana básica de sono preservado, não havendo necessidade de uso de um medicamento para insônia.

Tabela 3 - Tipos de Problemas Relacionados a Medicamentos encontrados nos pacientes em uso de carbonato de lítio atendidos na Unidade Básica de Saúde Aparecida / Caranazal

\begin{tabular}{|c|c|c|}
\hline TIPO DE PRM & OCORRÊNCIA & $(\%)$ \\
\hline \multicolumn{3}{|l|}{ NECESSIDADE } \\
\hline $\begin{array}{l}\text { PRM 01: Problema de saúde não } \\
\text { tratado. O problema de saúde está } \\
\text { relacionado ao fato de paciente não } \\
\text { receber a medicação que necessita. }\end{array}$ & - & - \\
\hline $\begin{array}{l}\text { PRM 02: Efeito de um medicamento } \\
\text { não necessário. O problema de saúde } \\
\text { do paciente está relacionado ao fato } \\
\text { dele fazer uso de uma medicação que } \\
\text { não necessita. }\end{array}$ & 01 & 10 \\
\hline \multicolumn{3}{|l|}{ EFETIVIDADE } \\
\hline $\begin{array}{l}\text { PRM 03: Inefetividade não quanti- } \\
\text { tativa da medicação. O problema de } \\
\text { saúde do paciente está relacionado } \\
\text { com a inefetividade do medicamento, } \\
\text { mas a inefetividade não está relacio- } \\
\text { nada com a quantidade da dose. }\end{array}$ & - & - \\
\hline $\begin{array}{l}\text { PRM 04: Inefetividade quantitativa } \\
\text { da medicação. O medicamento não é } \\
\text { efetivo por que o paciente utiliza dose } \\
\text { inferior que o necessário para tratar o } \\
\text { problema de saúde. }\end{array}$ & 09 & 90 \\
\hline \multicolumn{3}{|l|}{ SEGURANÇA } \\
\hline $\begin{array}{l}\text { PRM 05: Insegurança não quantita- } \\
\text { tiva da medicação. O medicamento } \\
\text { não é seguro, mas independe da } \\
\text { quantidade da dose. }\end{array}$ & - & - \\
\hline $\begin{array}{l}\text { PRM 06: Insegurança quantitativa } \\
\text { da medicação. O medicamento não é } \\
\text { seguro, estando diretamente ligada a } \\
\text { quantidade da dose. }\end{array}$ & - & - \\
\hline
\end{tabular}

Fonte: Dados da pesquisa

Apresenta-se ainda na Tabela 3 que 90\% (9/10) foram classificados quanto à efetividade em PRM 04 que destaca inefetividade quantitativa da medicação, classificação esta que pode responder aos resultados apresentados nas litemias analisadas onde $77,78 \%$ dos pacientes apresentam resultados abaixo da faixa terapêutica.

\section{DISCUSSÃO}

As características sócias demográficas dos pacientes estudados apresentadas na tabela 01 mostram que a média de idade dos pacientes acompanhados foi de 41,4 anos, dados que convergem com os resultados encontrados nos estudos de Lima (2005) e Huguet (2010), que destacaram média de idade próxima ao encontrado na presente pesquisa e ainda, como apresentado na pesquisa de Freire (2013), onde 91,1\% dos pesquisados apresentaram idade superior a 30 anos.

A prevalência em relação ao sexo foi de $60 \%(6 / 10)$ para o sexo masculino, no entanto tem-se referência que o Transtorno Depressivo Recorrente é duas vezes mais comum em mulheres adolescentes e adultas do que em adolescentes e adultos do sexo masculino como destacado no estudo de Freire (2013)que apontou uma prevalência de $78,6 \%$ para o sexo feminino de pacientes com Transtorno Bipolar do Humor, divergem também dos resultados encontrados nesta pesquisa, achados em um estudo de Huguet (2010), que mostra 39,6\% para homens e $60,4 \%$ para mulheres. Porém, destaca-se que no presente estudo não houve a classificação do tipo de THB se tipo I ou tipo II, ou seja, a amostra é de pacientes com diagnostico geral de THB e não classificado por tipo.

Com relação ao nível de escolaridade, foi observado no estudo que a maioria, $60 \%(6 / 10)$, dos pacientes pesquisados declararam ter completado o ensino médio, esse resultado se diferencia dos achados de Freire (2013) onde apenas 32,5\% apresentavam escolaridade semelhante.

Estudos de Baratto, Winter, e Falkenberg (2008), consideram que a educação para os pacientes pode melhorar a adesão ao tratamento, permitindo que recebam benefício máximo do tratamento e facilitando o ajuste à doença. Considera, ainda que a psicoeducação seja uma ferramenta útil na aceitação da doença, que faz com que os pacientes entendam seu significado e se tornem aptos a desenvolver papéis mais ativos e chegar a decisões mais seguras sobre seus tratamentos.

Vale destacar que $11,11 \%$ (01/09) declarou ter completado o nível superior achado que pode afirmar a pesquisa feito por Baratto, Winter, e Falkenberg (2008), pois nesta pesquisa este participante é o que apresenta melhor adesão ao tratamento que pode ser evidenciado no resultado da litemia, pois é o único pesquisado que encontra-se na faixa terapêutica, isto é, em eutimia o que é evidenciado na tabela 03 . 
A atual pesquisa também evidenciou esse dado, sendo a maioria de solteiros $90,0 \%$ (9/10), resultado que corrobora com estudo de Freire (2013), onde 51,8\% dos pesquisados declaram ser solteiros.

Apresentados na tabela 03 estão os resultados de dosagem de lítio realizados pelos pacientes em acompanhamento, destaca-se que apenas 09 /10 realizaram o exame no período da pesquisa, nos resultados $11,1 \%$ (01/09) está acima da faixa terapêutica caracterizado como risco para intoxicação de acordo com estudos de Goodman e Gilman (2006); Zung, Michelon \& Cordeiro (2010) que apontam níveis de litemia acima de 2,0 $\mathrm{mEq} / \mathrm{L}$ frequentemente relacionados com sinais clínicos de intoxicação podendo levar a exacerbação de efeitos colaterais; $11,1 \%(01 / 09)$ está dentro da faixa terapêutica representando uma boa adesão ao tratamento e 77, 78\% (07/09) estão abaixo da faixa terapêutica representando assim, baixo nível de adesão ao tratamento. Quanto à classificação dos Problemas Relacionados ao Medicamento 90\% (09/10) foram classificados conforme o Consenso de Granada (Comité de Consenso, 2002) quanto à efetividade em PRM 04 que destaca inefetividade quantitativa da medicação.

Quando o problema da terapia medicamentosa é demonstrado na dosagem de lítio muito baixa, Rovers (2010) indica as possíveis causas, que podem ser: dosagem incorreta, administração incorreta, interação medicamentosa, estocagem incorreta e duração inadequada da terapia medicamentosa.

\section{CONCLUSÕES}

De acordo com os resultados apresentados nesta pesquisa podemos destacar que na amostra estudada 77,8\% dos pacientes apresentaram litemia abaixo da faixa terapêutica recomentada pelo Ministério da Saúde, 11,1\% está em eutimia e $11,1 \%$ acima da faixa terapêutica, isto é, em risco de intoxicação. Estes resultados ressalta a importância do acompanhamento farmacoterapêutico de pacientes com diagnóstico de transtorno mental, especificamente o Transtorno Bipolar do Humor, devido a sua especificidade relacionada à necessidade da implementação da Atenção Farmacêutica aliada a Assistência de Enfermagem mais próxima ao paciente, na tentativa de identificar os problemas relacionados a medicamentos e o reconhecimento de interações medicamentosas em tempo hábil, a intervenção em saúde pela equipe multiprofissional, permitirá a avaliação quanto a adesão ao tratamento medicamentoso proposto e consequentemente melhorar qualidade de vida desses pacientes.

\section{REFERÊNCIAS}

Baratto, L., Winter, E., \& Falkenberg, M. (2008). Avaliação da adesão a farmacoterapia com litio e da percepção do tratamento medicamentoso entre pacientes bipolares do hospital universitário de Florianópolis. Latin American Journal of Pharmacy, 27(6),820-855.

Belmaker, R.H. (2004).Bipolar disorder.New England Journal of Medicine,351(5),476-486.doi: 10.1056/ NEJMra035354

Benazzi, F. (2007). Bipolar II disorder: Epidemiology, diagnosis and management. CNS Drugs, 21(9), 727740 .

Beyer, J., Kuchibhatla, M., Gersing, K., \& Krishnan, K.R. (2005). Medical comorbidity in a bipolar outpatient clinical population. Neuropsychopharmacology, 30(2),401-404.

Comité de Consenso. (2002). Segundo consenso de Granada sobre problemas relacionados com medicamentos. Ars Pharmaceutica, 43(3-4),175-184.I

Craig, C.R., \& Stitzel, R.E. (2005). Farmacologia Moderna. ( $6^{\mathrm{a}}$ ed.). Rio de Janeiro: Koogan.

De Meneses, A.L., \& Sá, M.L. (2010). Atenção farmacêutica ao idoso: Fundamentos e propostas. Journal of the Brazilian Society of Geriatrics and Gerontology, 4(3), 154-161.

Dourado, J.C.V. (2012). Efeitos colaterais dermatológicos e renais carbonato de lítio: revisão de literatura. Universidade Federal da Bahia, Salvador, Bahia.

Figueiredo, A.L., Souza, L., Dell'Áglio Jr., J.C., \& Argimon, I.I.L. (2009). Uso da psicoeducação no tratamento do transtorno bipolar. Revista Brasileira de Terapia Comportamental Cognitiva, 11(1),15-24.

Freire, E. C. (2013). Adesão ao tratamento medicamentoso em usuários com transtorno do humor de centro de atenção psicossocial do nordeste do Brasil. Revista Ciências Farmacêutica Básica Aplicada, 34(4),565-570.

Gajwani, P. (2009). Treatment-refractory bipolar disorder: Classification to aid in clinical management. Expert Opinionon Pharmacotherapy,10(12),1907-1915. 
